\title{
Influence of Plasma Waves on the Photoacoustic Signal of Silicon Samples
}

\author{
Lukasz Chrobak • Miroslaw Malinski • \\ Aleksy Patryn
}

Received: 9 December 2008 / Accepted: 21 June 2011 / Published online: 7 July 2011 (C) The Author(s) 2011. This article is published with open access at Springerlink.com

\begin{abstract}
This article presents the results of theoretical and experimental studies of the influence of plasma waves on the photoacoustic signals of Si samples. The aim of these investigations was to find and examine the sensitive measurement configurations and the configurations of the sample in the photoacoustic cell for detection and measurement of the lifetime of excess carriers. Two measurement configurations were examined: transmission and phase lag and two configurations of the sample in the photoacoustic cell. This article demonstrates that both configurations for samples with differently prepared surfaces are effective for observation and detection of plasma waves with the photoacoustic method.
\end{abstract}

Keywords Photoacoustics $\cdot$ Plasma wave $\cdot$ Silicon

\section{Introduction}

The photoacoustic (PA) signal in semiconductors is a result of three irradiative processes: intraband thermalization of carriers, diffusion of carriers followed by their volume recombination, and the surface recombination of excess carriers on both surfaces of the sample. These three processes generate three spatial temperature distributions in the sample. The theory of the plasma wave effect has been developed since

\footnotetext{
L. Chrobak $(\varangle) \cdot$ M. Malinski · A. Patryn

Department of Electronics and Computer Studies, Technical University of Koszalin, 2 Śniadeckich St., 75-453 Koszalin, Poland e-mail: lukasz.chrobak@tu.koszalin.pl

M. Malinski

e-mail: miroslaw.malinski@tu.koszalin.pl
}

A. Patryn
e-mail: aleksy.patryn@tu.koszalin.pl 
the 1980s [1,2]. The influence of the plasma wave effect on the photoacoustic signal was also investigated by Dramicanin et al. [3] and applied for Ge samples. This effect was also described by Todorovic and Nikolic [4] for Ge crystals. The plasma wave effect observed for CdTe/glass system was reported by Bernal-Alvarado et al. [5]. The influence of the plasma wave effect on a photoacoustic signal of a polycrystal CdTe/glass system was also investigated by Martin et al. [6].

The plasma wave effect for $\mathrm{CdInGaS}_{4}$ crystal samples was described by Shen and Toyoda [7]. Thermal and electronic transport properties obtained by the PA method for $\mathrm{Yba}_{2} \mathrm{Cu}_{3} \mathrm{O}_{7-\mathrm{x}}$ and $\mathrm{InBi}$ crystals were described in [8] and [9], respectively. Results of the investigations of ion-modified Si by the PA method were also presented in [10-12]. Thin layers of CdTe on a glass substrate were investigated by Bernal-Alvarado and Vargas-Luna [13]. A contribution of carrier transport processes to the photoacoustic effect in doped narrow gap semiconductors $\mathrm{PbTe}, \mathrm{PbSe}$, and $\mathrm{SnTe}$ was described by Radulovic et al. [14]. The theoretical model that was used for computations of the PA characteristics in this article is called a compact model and was described previously [15]. The influence of plasma waves on the PA microphone and piezoelectric spectra of semiconductors was investigated theoretically and described in [16].

For plasma wave-involved PA experiments, different approaches, i.e., experimental configurations and samples were applied and described in the literature. For samples with equally prepared surfaces (e.g., polished), the difference of the PA phase frequency characteristics, or the ratio of the amplitudes, obtained for different thickness of the samples, were described in the literature $[3,4]$. The most frequently used configuration for the measurement of the plasma wave contribution to the PA signal was the transmission configuration and the measurements of the phase frequency characteristics.

\section{Experimental Procedure}

For investigations of the influence of the plasma wave effect on the PA signal presented in this article, the transmission and the phase-lag measurement configurations were chosen. The Si wafers had two differently prepared sides: the polished side and the roughened side. The polished side was optically of a mirror type while the roughened side was matt. For the purpose of determination of the lifetime of excess carriers in silicon samples, PA signals were measured for two configurations of the samples in the photoacoustic cell. In the first configuration, the polished side of the sample was illuminated while in the second configuration the roughened side was illuminated. The microphone detection of the photoacoustic signal was applied with a phase selective measurement with the lock-in amplifier SR830. For generation of the PA signal, the intensity-modulated beam of the $532 \mathrm{~nm}$ laser of the $50 \mathrm{~mW}$ optical power was applied. Measurements were performed in the range of frequencies of modulation from $f=30 \mathrm{~Hz}$ to $f=1000 \mathrm{~Hz}$. The electret microphone with a low noise preamplifier was used for detection of the PA signal. The diameter of the applied laser beam was smaller than $0.5 \mathrm{~mm}$. A schematic diagram of the experimental setup used in the measurements is presented in Fig. 1. 


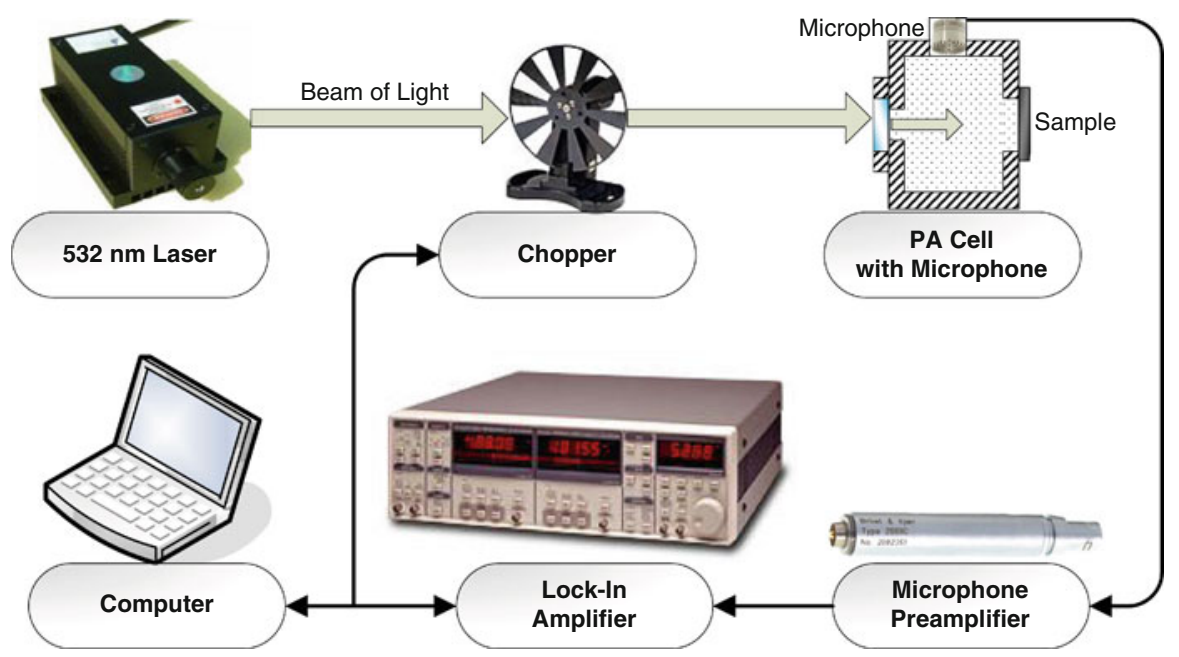

Fig. 1 Schematic diagram of the experimental setup used in the measurements

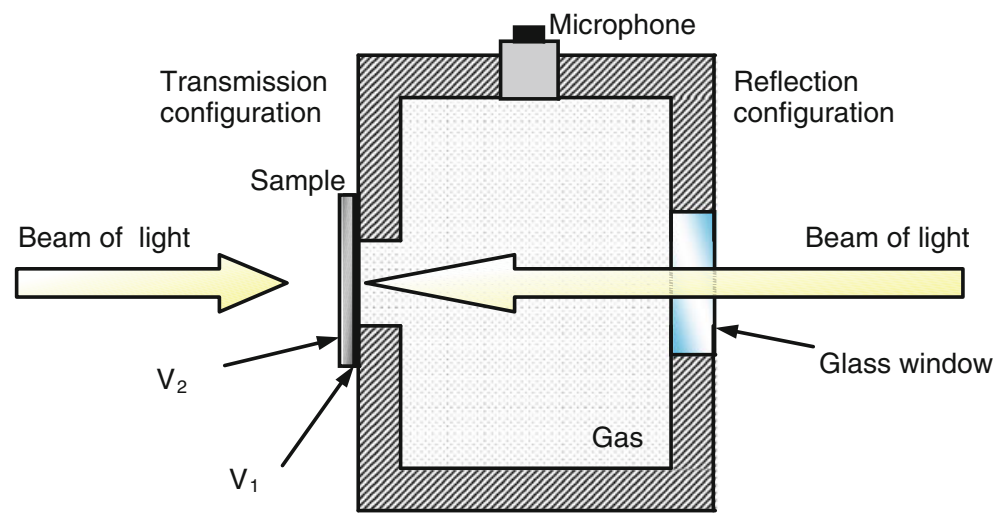

Fig. 2 Schematic diagram of the applied measurement configurations: in the phase transmission and in the phase-lag methods

A schematic diagram of the samples in the PA cell in the phase transmission and phase-lag configurations are presented in Fig. 2. In the transmission configuration, the sample was always illuminated from the left and it was illuminated first on the polished side and then turned over to illuminate the matt side. The PA signal was measured by the microphone. The difference of the phase frequency characteristics measured in these two transmission configurations of the sample in the PA cell was analyzed. In the phase-lag configuration, the sample was illuminated from the left and next from the right side and the PA signal was measured and the difference of the phase frequency characteristics was analyzed. For determination of the thermal diffusivity, the roughened side of the sample was illuminated from the left and from the right and again the difference of the phase frequency characteristics was analyzed. 


\section{Theoretical Considerations}

The idea of the approach presented in this article is the following. In the measurements, the transmission and phase-lag configurations were applied as the sensitivity of these configurations for the plasma waves contribution to the PA signal was expected to be high. Model simulations performed, in a plasma wave model, for different sample configurations in the PA cell and with differently prepared surfaces of a sample, indicated that both approaches analyzed in the article are comparatively sensitive for detection of plasma waves.

The transmission configuration means that one side of the sample is illuminated but the temperature of the other side of the sample is measured by the microphone as the periodical overpressure in the photoacoustic cell. The reflection configuration means that one side of the sample is illuminated and the temperature of the same side of the sample is measured by the microphone as the periodical overpressure in the photoacoustic cell. The value of the lifetime of excess carriers was extracted from the difference of the phase frequency characteristics of the PA signals measured in two configurations of the sample in the PA cell, i.e., when a polished or the roughened side of the sample was illuminated.

The temperature spatial distribution in the sample, arising as a result of its illumination by the intensity-modulated beam of light, is in general a function of several physical parameters and is described below.

As some variables are constant (i.e., $d, R, \alpha, E, E_{\mathrm{g}}, \beta$ ), they will not be included in further considerations.

$$
\begin{aligned}
T\left(x, f, \tau, D, V_{1}, V_{2}\right)= & \Theta_{T}(x, f)+\Theta_{\mathrm{NRR}}\left(x, f, \tau, D, V_{1}, V_{2}\right) \\
& +\Theta_{\mathrm{SR}}\left(x, f, \tau, D, V_{1}, V_{2}\right)
\end{aligned}
$$

where $\Theta_{T}(x, f)$ is the spatial temperature distribution being the result of the intraband thermalization of carriers, $\Theta_{\mathrm{NRR}}\left(x, f, \tau, D, V_{1}, V_{2}\right)$ is the spatial temperature distribution being the result of the volume nonradiative recombination of carriers, and $\Theta_{\mathrm{SR}}\left(x, f, \tau, D, V_{1}, V_{2}\right)$ is the spatial temperature distribution being the result of the surface recombination of carriers.

The above spatial temperature distributions can be computed according to the following formulae:

$$
\begin{aligned}
\sigma(f) & =(1+\mathrm{i})\left(\frac{\pi f}{\alpha}\right)^{\frac{1}{2}} \\
L e(f, \tau) & =\left[\frac{D \tau}{1+\mathrm{i} \omega(f) \tau}\right]^{\frac{1}{2}} \\
K(f, \tau) & =\frac{I_{0}}{2 E D} \frac{\beta}{\beta^{2}-[L e(f, \tau)]^{-2}} \\
\varepsilon_{\mathrm{b}}\left(f, \tau, V_{2}\right) & =\frac{D}{V_{2} L e(f, \tau)} \quad \varepsilon_{\mathrm{g}}\left(f, \tau, V_{1}\right)=\frac{D}{V_{1} \operatorname{Le}(f, \tau)} \\
\eta_{\mathrm{b}}\left(f, \tau, V_{2}\right) & =\varepsilon_{\mathrm{b}}\left(f, \tau, V_{2}\right) \beta L e(f, \tau) \quad \eta_{\mathrm{g}}\left(f, \tau, V_{1}\right)=\varepsilon_{\mathrm{g}}\left(f, \tau, V_{1}\right) \beta \operatorname{Le}(f, \tau)
\end{aligned}
$$




$$
\begin{aligned}
& B_{1}\left(f, \tau, D, V_{1}, V_{2}\right) \\
& =\frac{\left[\eta_{\mathrm{g}}\left(f, \tau, V_{1}\right)+1\right]\left[\varepsilon_{\mathrm{b}}\left(f, \tau, V_{2}\right)-1\right] \mathrm{e}^{\frac{-d}{L e(f, \tau)}}-\left[\eta_{\mathrm{b}}\left(f, \tau, V_{2}\right)-1\right]\left[\varepsilon_{\mathrm{g}}\left(f, \tau, V_{1}\right)+1\right] \mathrm{e}^{-\beta d}}{\left[\varepsilon_{\mathrm{g}}\left(f, \tau, V_{1}\right)+1\right]\left[\varepsilon_{\mathrm{b}}\left(f, \tau, V_{2}\right)+1\right] \mathrm{e}^{\frac{d}{L e(f, \tau)}}-\left[\varepsilon_{\mathrm{g}}\left(f, \tau, V_{1}\right)-1\right]\left[\varepsilon_{\mathrm{b}}\left(f, \tau, V_{2}\right)-1\right] \mathrm{e}^{\frac{-d}{L e(f, \tau)}}}
\end{aligned}
$$

$B_{2}\left(f, \tau, D, V_{1}, V_{2}\right)$

$$
=\frac{\left[\eta_{\mathrm{g}}\left(f, \tau, V_{1}\right)+1\right]\left[\varepsilon_{\mathrm{b}}\left(f, \tau, V_{2}\right)+1\right] \mathrm{e}^{\frac{d}{\operatorname{Le}(f, \tau)}}-\left[\eta_{\mathrm{b}}\left(f, \tau, V_{2}\right)-1\right]\left[\varepsilon_{\mathrm{g}}\left(f, \tau, V_{1}\right)-1\right] \mathrm{e}^{-\beta d}}{\left[\varepsilon_{\mathrm{g}}\left(f, \tau, V_{1}\right)+1\right]\left[\varepsilon_{\mathrm{b}}\left(f, \tau, V_{2}\right)+1\right] \mathrm{e}^{\frac{d}{\operatorname{Le}(f, \tau)}}-\left[\varepsilon_{\mathrm{g}}\left(f, \tau, V_{1}\right)-1\right]\left[\varepsilon_{\mathrm{b}}\left(f, \tau, V_{2}\right)-1\right] \mathrm{e}^{\frac{-d}{\operatorname{Le}(f, \tau)}}}
$$

$$
\begin{aligned}
\delta n(x)= & K(f, \tau)\left[B_{1}\left(f, \tau, D, V_{1}, V_{2}\right) \mathrm{e}^{\frac{x}{L e(f, \tau)}}\right. \\
& \left.+B_{2}\left(f, \tau, D, V_{1}, V_{2}\right) \mathrm{e}^{\frac{-x}{L e(f, \tau)}}-\mathrm{e}^{-\beta x}\right]
\end{aligned}
$$

$$
W_{1}(z, x)=\mathrm{e}^{-\sigma(x-z)}+\mathrm{e}^{-\sigma(2 d-z-x)}+\mathrm{e}^{-\sigma(z+x)}+\mathrm{e}^{-\sigma(2 d+z-x)}
$$

$$
W_{2}(z, x)=\mathrm{e}^{-\sigma(z-x)}+\mathrm{e}^{-\sigma(z+x)}+\mathrm{e}^{-\sigma(2 d-z-x)}+\mathrm{e}^{-\sigma(2 d-z+x)}
$$

$$
\begin{aligned}
\Theta_{\mathrm{T}}(x, f)= & \frac{\left(E-E_{\mathrm{g}}\right) I_{0} \beta}{2 E \kappa \sigma\left(1-\mathrm{e}^{-2 \sigma d}\right)}\left[\int_{0}^{x} \mathrm{e}^{-\beta z} W_{1}(z, x) \mathrm{d} z\right. \\
& \left.+\int_{x}^{d} \mathrm{e}^{-\beta z} W_{2}(z, x) \mathrm{d} z\right]
\end{aligned}
$$

$\Theta_{\mathrm{NRR}}\left(x, f, \tau, D, V_{1}, V_{2}\right)=\frac{E_{\mathrm{g}} I_{0}}{\kappa \sigma \tau\left(1-\mathrm{e}^{-2 \sigma d}\right)}\left[\int_{0}^{x} \delta n(z) W_{1}(z, x) \mathrm{d} z\right.$

$$
\left.+\int_{x}^{d} \delta n(z) W_{2}(z, x) \mathrm{d} z\right]
$$

$$
\begin{aligned}
\Theta_{\mathrm{SR}}\left(x, f, \tau, D, V_{1}, V_{2}\right)= & \frac{E_{\mathrm{g}} I_{0}}{\kappa \sigma\left(1-\mathrm{e}^{-2 \sigma d}\right)}\left[V_{1} \delta n(0) W_{1}(0, x)\right. \\
& \left.+V_{2} \delta n(d) W_{2}(d, x)\right]
\end{aligned}
$$

where $x$ is the spatial coordinate along the thickness of the sample normal to the surface, $z$ is the integration variable, $f$ is the frequency of modulation of the intensity of the beam of light, $d$ is the thickness of the sample, $R$ is the thermal reflection coefficient between the sample and the backing, $\alpha$ is the thermal diffusivity of the sample, $E$ is the energy of photons of the illuminating light, $E_{\mathrm{g}}$ is the energy gap value of a semiconductor, $D$ is the diffusion coefficient of carriers, $\beta$ is the optical absorption coefficient of the semiconductor for the illuminating beam of light, $\tau$ is the lifetime of excess carriers, $V_{1}, V_{2}$ are velocities of surface recombination of carriers on both sides of the sample: illuminated and dark side, respectively, $\delta n(x)$ is the spatial distribution 
of the concentration of excess carriers, $I_{0}$ is the intensity of light, and $\kappa$ is the thermal conductivity.

The difference of the phase frequency characteristics investigated in this work in the transmission configuration can be expressed as

$$
\begin{aligned}
\Delta P_{\mathrm{PD}}\left(d, f, \tau, D, V_{1}, V_{2}\right)= & \frac{180}{\pi} \arg \left[\frac{T\left(d, f, \tau, D, V_{2}, V_{1}\right)}{\sigma(f)}\right] \\
& -\frac{180}{\pi} \arg \left[\frac{T\left(d, f, \tau, D, V_{1}, V_{2}\right)}{\sigma(f)}\right]
\end{aligned}
$$

The difference of the phase frequency characteristics investigated in this work in the phase-lag configuration can be expressed as

$$
\begin{aligned}
\Delta P_{\mathrm{PL}}\left(0, d, f, \tau, D, V_{1}, V_{2}\right)= & \frac{180}{\pi} \arg \left[\frac{T\left(0, f, \tau, D, V_{1}, V_{2}\right)}{\sigma(f)}\right] \\
& -\frac{180}{\pi} \arg \left[\frac{T\left(d, f, \tau, D, V_{2}, V_{1}\right)}{\sigma(f)}\right]
\end{aligned}
$$

The example theoretical phase difference characteristics in the transmission configuration showing dependencies of the PA phase differences on the lifetime of carriers obtained for different frequencies of modulation, thickness of the sample $d=0.058 \mathrm{~cm}, D=15 \mathrm{~cm}^{2} \cdot \mathrm{s}^{-1}, V_{1}=1500 \mathrm{~cm} \cdot \mathrm{s}^{-1}$, and $V_{2}=100 \mathrm{~cm} \cdot \mathrm{s}^{-1}$ are presented in Fig. 3.

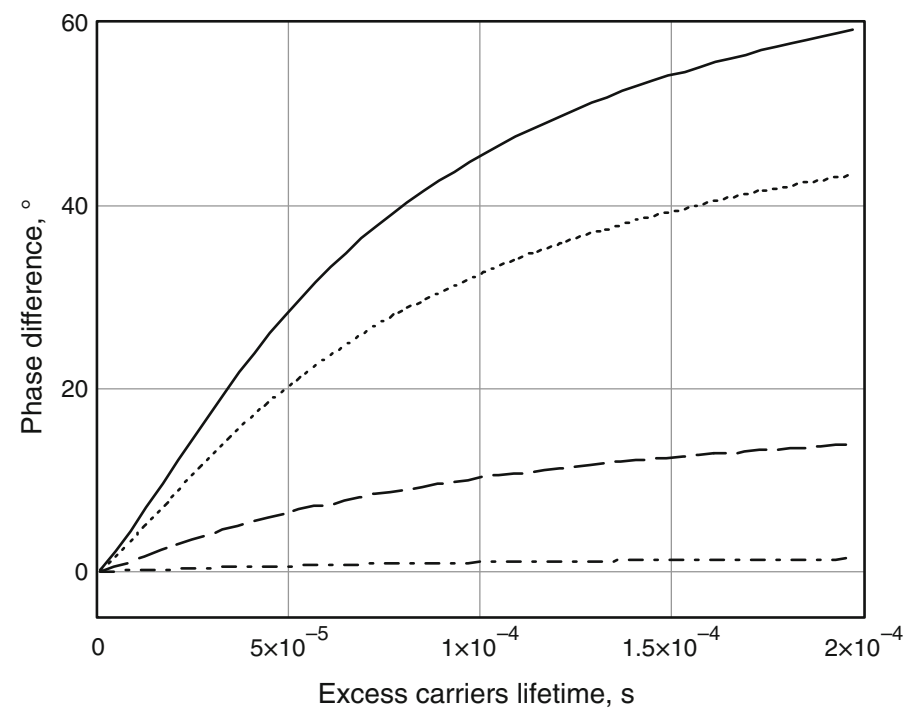

Fig. 3 Theoretical dependencies of the phase difference of the PA signals in the transmission configuration on the lifetime of carriers computed for: $d=0.058 \mathrm{~cm}, \alpha=0.95 \mathrm{~cm}^{2} \cdot \mathrm{s}^{-1}, E=2.0 \mathrm{eV}, f=300 \mathrm{~Hz}$ (solid), $200 \mathrm{~Hz}$ (dotted), $100 \mathrm{~Hz}$ (dashed), $20 \mathrm{~Hz}$ (dashed-dotted), $D=15 \mathrm{~cm}^{2} \cdot \mathrm{s}^{-1}, V_{1}=1500 \mathrm{~cm} \cdot \mathrm{s}^{-1}$, $V_{2}=100 \mathrm{~cm} \cdot \mathrm{s}^{-1}$ 


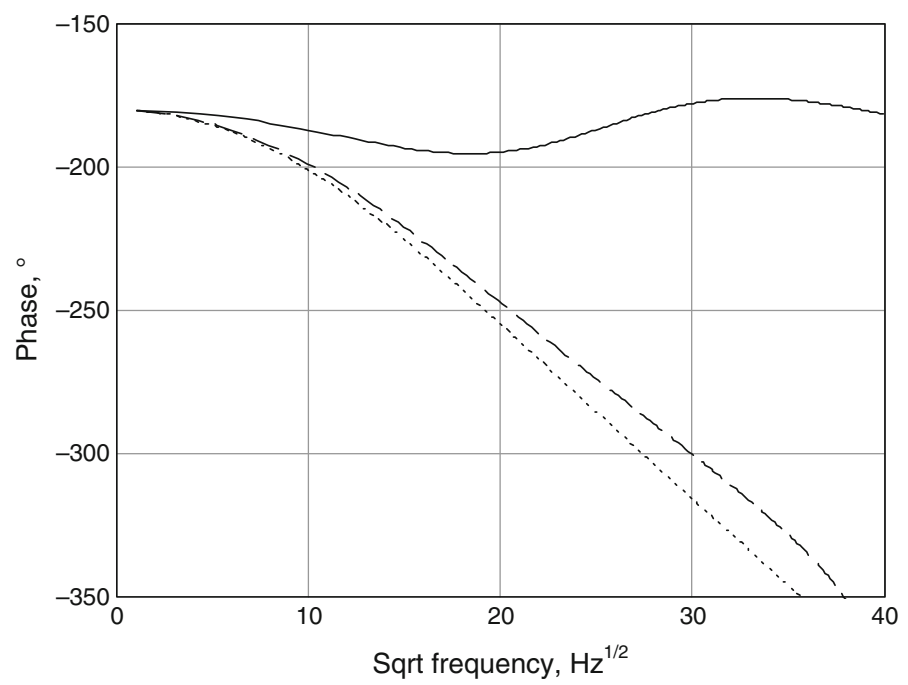

Fig. 4 Theoretical characteristics of the phase of the PA signal versus the frequency of modulation in the transmission configuration computed for the parameters $\alpha=0.95 \mathrm{~cm}^{2} \cdot \mathrm{s}^{-1}, \tau=0.140 \times 10^{-3} \mathrm{~s}$, $d=0.058 \mathrm{~cm}, \beta=10^{3} \mathrm{~cm}^{-1}, D=15 \mathrm{~cm}^{2} \cdot \mathrm{s}^{-1}$ and different velocities of surface recombination: $V_{1}=1500 \mathrm{~cm} \cdot \mathrm{s}^{-1}, V_{2}=100 \mathrm{~cm} \cdot \mathrm{s}^{-1}$ (dashed line) and $V_{1}=100 \mathrm{~cm} \cdot \mathrm{s}^{-1}, V_{2}=1500 \mathrm{~cm} \cdot \mathrm{s}^{-1}$ (solid line). Dotted line phase of the intra-band thermalization component only

The characteristics presented above show that the expected phase difference increases with increasing modulation frequency and with increasing excess carrier lifetime.

The idea of the phase difference transmission measurements is presented in Figs. 4, 5, and 6. The solid lines show the phase of the PA signal, measured in the transmission configuration, when a polished side of the sample is illuminated. Dashed lines show the phase of the PA signal, measured in the same configuration, but when the roughened side of the sample is illuminated.

In Figs. 4, 5, and 6, the dotted lines are the phase characteristics of the intra-band thermalization component only. As seen from the figures, in this second case the phase of the PA signal is practically close to the phase of the intra-band component only. In the experiment, the difference of these two characteristics (solid and dashed lines) was investigated. The lifetime of the carriers was extracted from the fitting of theoretical curves to experimental phase difference frequency characteristics.

It results from the figures above that when the lifetime of carriers is small, i.e., smaller than a few microseconds, then the phase difference of the PA signals, at least in the frequency range up to $1000 \mathrm{~Hz}$, disappears. For longer lifetimes, the difference between the phase frequency characteristics (solid and dashed lines) appears. A longer lifetime results in a larger difference between the actual phase and the phase of the intraband thermalization component.

The differences visible in Figs. 4 and 5 between solid and dashed lines, when illuminating the polished or roughened surface, are the result of competition of two processes, i.e., diffusion of carriers and surface recombination. 


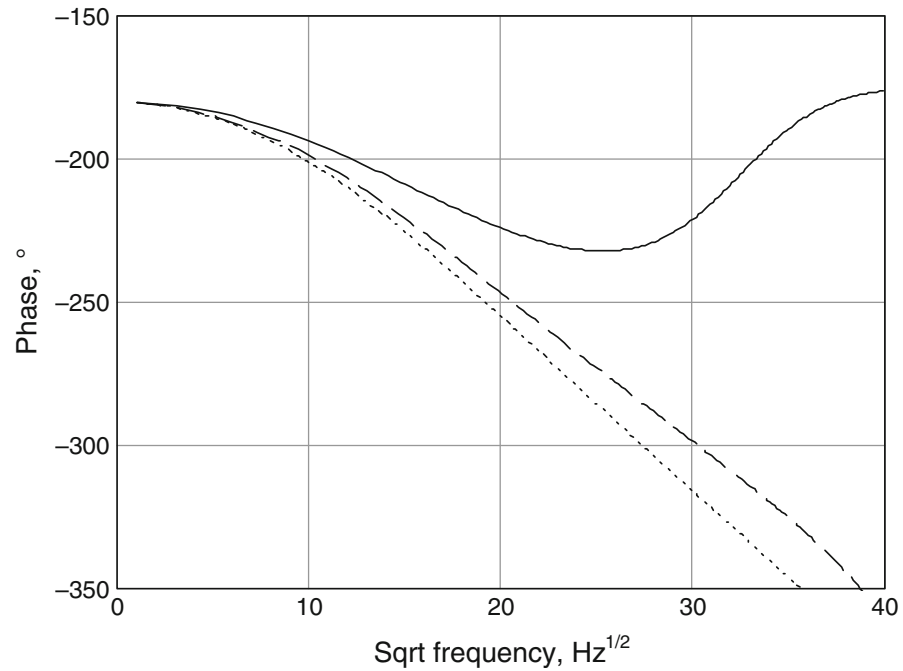

Fig. 5 Theoretical characteristics of the phase of the PA signal versus the frequency of modulation in the transmission configuration computed for the parameters $\alpha=0.95 \mathrm{~cm}^{2} \cdot \mathrm{s}^{-1}, \tau=0.050 \times 10^{-3} \mathrm{~s}$, $d=0.058 \mathrm{~cm}, \beta=10^{3} \mathrm{~cm}^{-1}, D=15 \mathrm{~cm}^{2} \cdot \mathrm{s}^{-1}$ and different velocities of surface recombination: $V_{1}=1500 \mathrm{~cm} \cdot \mathrm{s}^{-1}, V_{2}=100 \mathrm{~cm} \cdot \mathrm{s}^{-1}$ (dashed line) and $V_{1}=100 \mathrm{~cm} \cdot \mathrm{s}^{-1}, V_{2}=1500 \mathrm{~cm} \cdot \mathrm{s}^{-1}$ (solid line). Dotted line phase of the intra-band thermalization component only

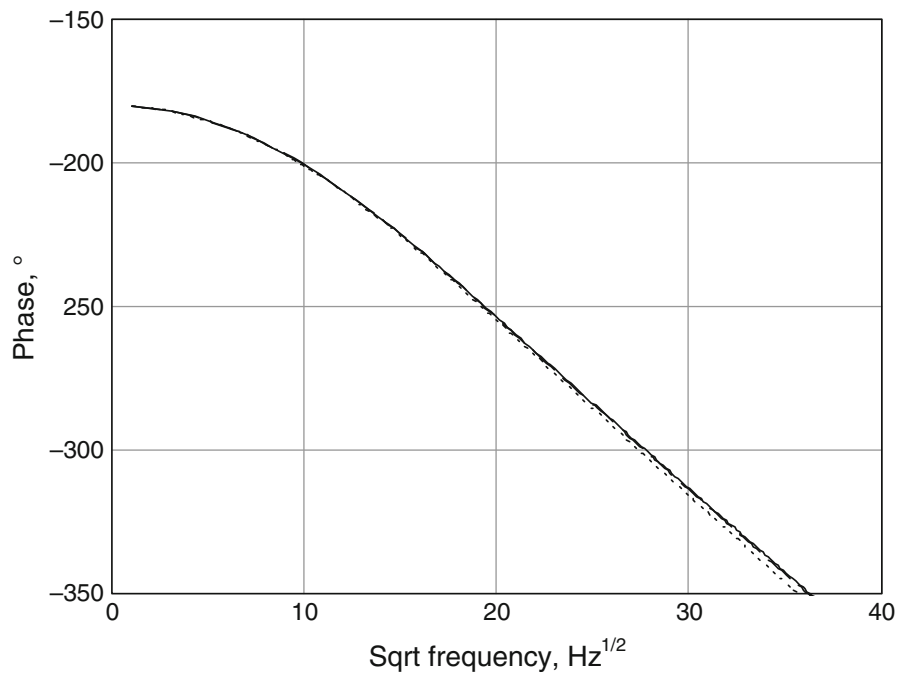

Fig. 6 Theoretical characteristics of the phase of the PA signal versus the frequency of modulation in the transmission configuration, computed for the parameters $\alpha=0.95 \mathrm{~cm}^{2} \cdot \mathrm{s}^{-1}, \tau=0.001 \times 10^{-3} \mathrm{~s}$, $d=0.058 \mathrm{~cm}, \beta=10^{3} \mathrm{~cm}^{-1}, D=15 \mathrm{~cm}^{2} \cdot \mathrm{s}^{-1}$ and different velocities of surface recombination: $V_{1}=1500 \mathrm{~cm} \cdot \mathrm{s}^{-1}, V_{2}=100 \mathrm{~cm} \cdot \mathrm{s}^{-1}$ (dashed line) and $V_{1}=100 \mathrm{~cm} \cdot \mathrm{s}^{-1}, V_{2}=1500 \mathrm{~cm} \cdot \mathrm{s}^{-1}$ (solid line). Dotted line phase of the intra-band thermalization component only 


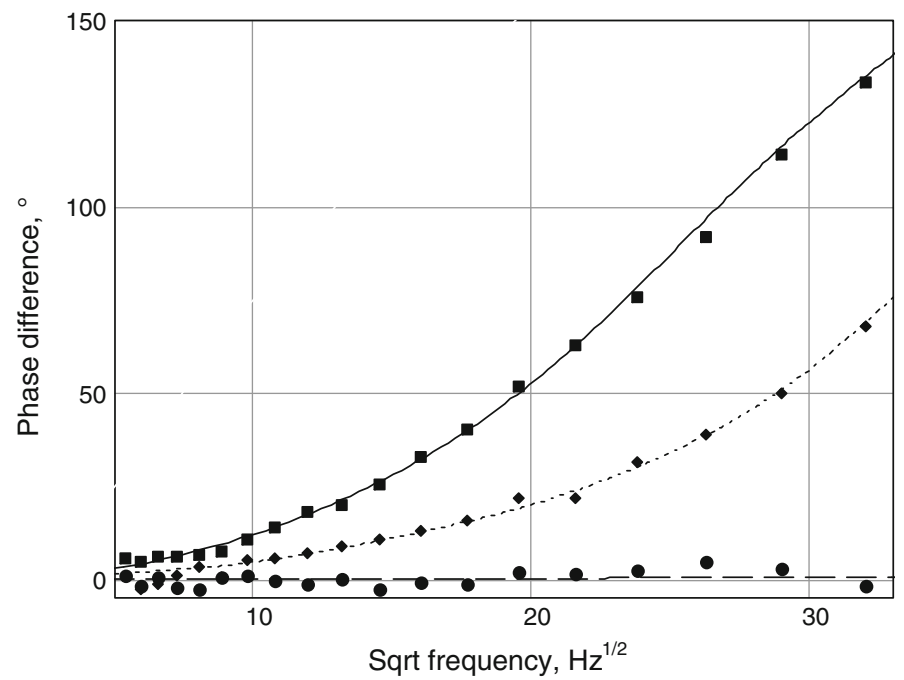

Fig. 7 Experimental and theoretical phase difference characteristics of the silicon samples in the transmission measurement configuration. Squares: $\tau=0.140 \times 10^{-3} \mathrm{~s}, d=0.058 \mathrm{~cm}, L=0.046 \mathrm{~cm}$, diamonds: $\tau=0.05 \times 10^{-3} \mathrm{~s}, d=0.049 \mathrm{~cm}, L=0.027 \mathrm{~cm}$, circles: $\tau=0.001 \times 10^{-3} \mathrm{~s}, d=0.075 \mathrm{~cm}$, $L=0.0038 \mathrm{~cm}, \alpha=0.95 \mathrm{~cm}^{2} \cdot \mathrm{s}^{-1}, E=2.0 \mathrm{eV}, V_{1}=1500 \mathrm{~cm} \cdot \mathrm{s}^{-1}, V_{2}=100 \mathrm{~cm} \cdot \mathrm{s}^{-1}$

In conclusion, the difference between the phase characteristics (solid and dashed lines) gives information about the lifetime of the carriers.

\section{Experimental Results}

The measured PA phase difference characteristics in the transmission configuration with the two configurations of the sample in the PA cell are presented in Fig. 7. Lifetimes of carriers were determined assuming the diffusion coefficient of carriers of $p$-type silicon $D=15 \mathrm{~cm}^{2} \cdot \mathrm{s}^{-1}$. The diffusion length of carriers can be determined as $L=(D \tau)^{1 / 2}$. The theoretical curves in Fig. 7 are differences of the phase characteristics (solid and dashed lines) presented in Figs. 4, 5, and 6, but computed for appropriate lifetimes of excess carriers as a result of the fitting procedure.

The experimental and theoretical characteristics obtained for the same samples but in the phase-lag measurement configuration are presented in Fig. 8.

The extracted values, from the multi-fitting procedure, of investigated samples have been presented in Table 1.

The PA characteristics are very complicated for interpretation as they are multiparameter functions when some of the parameters are unknown. To check the correctness of conclusions drawn from the PA experiments, the photocurrent (PC) experiment was performed.

Lifetimes of excess carriers of the same samples were determined from the modified Stevenson's photocurrent method when the lifetime can be determined from the dependence of the phase of the photocurrent versus the frequency of modulation of 


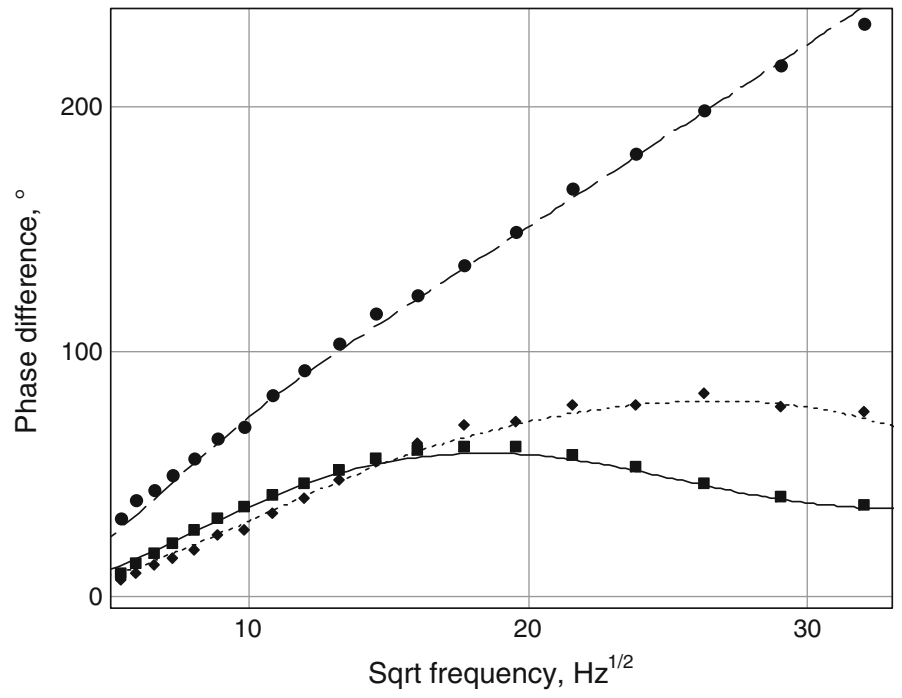

Fig. 8 Phase-lag frequency theoretical and experimental characteristics obtained for silicon samples. Squares: $\tau=0.140 \times 10^{-3} \mathrm{~s}, d=0.058 \mathrm{~cm}, L=0.046 \mathrm{~cm}$, diamonds: $\tau=0.050 \times 10^{-3} \mathrm{~s}$, $d=0.049 \mathrm{~cm}, L=0.027 \mathrm{~cm}$, circles: $\tau=0.001 \times 10^{-3} \mathrm{~s}, d=0.075 \mathrm{~cm}, L=0.0038 \mathrm{~cm}, \alpha=$ $0.95 \mathrm{~cm}^{2} \cdot \mathrm{s}^{-1}, E=2.0 \mathrm{eV}, V_{1}=1500 \mathrm{~cm} \cdot \mathrm{s}^{-1}, V_{2}=100 \mathrm{~cm} \cdot \mathrm{s}^{-1}$

Table 1 Values of adjustable parameters for the silicon samples obtained by the multifitting procedure

\begin{tabular}{lllllll}
\hline Sample no. & $d(\mathrm{~cm})$ & $\alpha\left(\mathrm{cm}^{2} \cdot \mathrm{s}^{-1}\right)$ & $D\left(\mathrm{~cm}^{2} \cdot \mathrm{s}^{-1}\right)$ & $V_{1}\left(\mathrm{~cm} \cdot \mathrm{s}^{-1}\right)$ & $V_{2}\left(\mathrm{~cm} \cdot \mathrm{s}^{-1}\right)$ & $\tau(\mathrm{s})$ \\
\hline 1 & 0.058 & 0.95 & 15 & 1408 & 150.34 & $140.0 \times 10^{-6}$ \\
2 & 0.049 & 0.95 & 15 & 1500 & 149.99 & $50.82 \times 10^{-6}$ \\
3 & 0.075 & 0.95 & 15 & 1499 & 150.19 & $1.37 \times 10^{-6}$ \\
\hline
\end{tabular}

the illuminating beam of light and when the polished side of the sample is illuminated. The experimental and theoretical PC characteristics are presented in Fig. 9. The phase of the photocurrent is the function of the lifetime as $\phi=\arctan (-\omega \tau)$.

From the comparison of the results of lifetimes determined by the two methods, $\mathrm{PA}$ and PC, one can draw a conclusion that the values are similar. The differences are the result of the simplicity of the RC model applied for the interpretation of the PC characteristics which is correct only for the samples exhibiting a perfect quality of the illuminated side of the sample, i.e., $V_{1}, V_{2} \rightarrow 0$. The lifetimes extracted from the PA characteristics depend, on the other hand, on the velocity of recombination of carriers, especially on the roughened side of the sample.

\section{Conclusions}

Results of computations and measurements presented in this article indicated that the influence of plasma waves is visible on the PA characteristics in a different way 


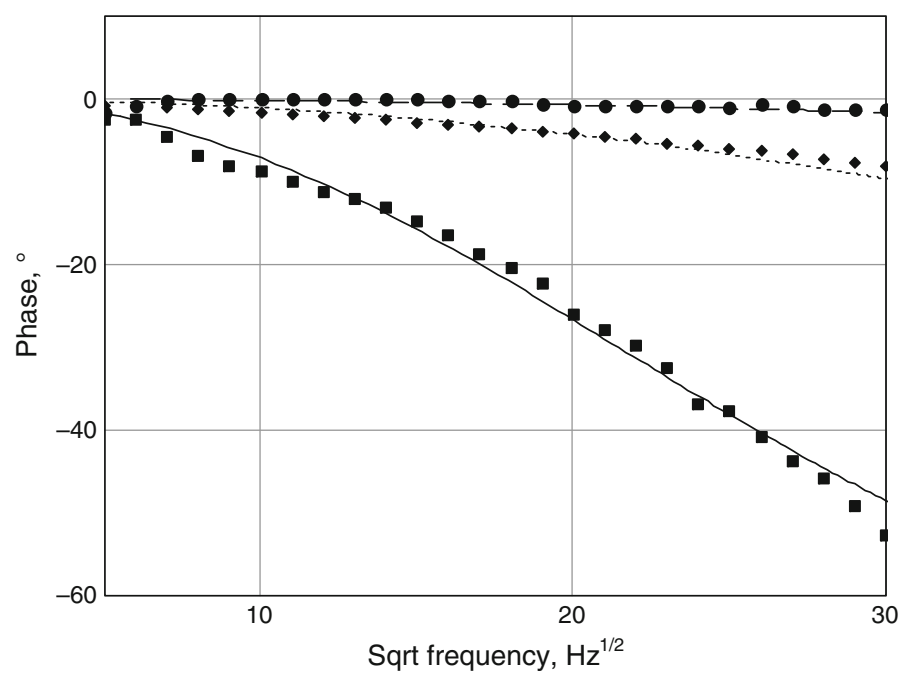

Fig. 9 Dependencies of the phase of the photocurrent on the frequency of modulation of the illuminating beam of light: circles: $\tau \sim 5 \mu \mathrm{s}$, diamonds: $\tau \sim 35 \mu \mathrm{s}$, squares: $\tau \sim 180 \mu \mathrm{s}$

depending on the configuration of the sample in the PA cell, the state of its surface, and the measurement configuration applied. It turned out that there are two sensitive PA measurement configurations for observation of the plasma wave contributions and the configuration of the sample in the PA cell when the polished side of the sample is illuminated and the roughened side is in the PA cell and when the roughened side is illuminated and the polished side is in the PA cell. The lifetime of carriers can be determined from the difference of the two PA phase transmission characteristics. The same results of the lifetimes were obtained in the phase-lag method when the polished side was illuminated in the transmission configuration and the roughened side was illuminated in the reflection configuration. The phase difference in both methods is a measure of the capability of evaluation of the lifetime of the excess carriers. In this sense, we can say that the phase-lag method is more sensitive than the phase difference in a transmission configuration, as it gives a bigger phase difference for the same set of material parameters and the same frequency of modulation. In general, this article shows how the optically generated excess carriers diffusing from the surface of the sample, where they are generated, to the volume of the sample and the opposite side of the sample, where they relax irradiatively, influence and modify the frequency phase photoacoustic characteristics measured in the experiment in the two presented configurations. This influence is caused by the fact that the place of generation and the place of irradiative relaxation of excess carriers are different. These places of relaxation can be modified by appropriate surface preparation modifying the velocities of the surface recombination of carriers. Without the diffusion process of optically generated carriers, the phase difference characteristics, analyzed in this article, would not have been observed. Samples with differently prepared surfaces, i.e., polished or roughened and exhibiting a short lifetime of carriers did not show the phase differences in the analyzed configurations. The results of the PA experiments presented in this article 
were confirmed by the measurements of the kinetics of the photocurrent giving similar values, in a reasonable agreement, of the lifetimes of excess carriers. The inaccuracies of lifetimes determined from the PA experiments are the result of the fact that the PA signal is a function of several parameters and some of them are not known exactly.

Acknowledgments This research work was financed from the budget funds for science of the Ministry of Science and Higher Education in the years 2010-2012 as a research project No. N N515604339. The authors, with hope for further cooperation, would like to thank Dr. Nikolai Abrosimov (Institute of Crystal Growth, Max-Born-Str. 2, D-12469 Berlin, Germany) for especially prepared silicon samples.

Open Access This article is distributed under the terms of the Creative Commons Attribution Noncommercial License which permits any noncommercial use, distribution, and reproduction in any medium, provided the original author(s) and source are credited.

\section{References}

1. L.C. Miranda, Appl. Opt. 21, 2923 (1982)

2. V.A. Sablikov, V.B. Sandomirskii, Phys. Status Solidi B 120, 471 (1983)

3. M.D. Dramicanin, Z.D. Ristovski, P.M. Nicolic, D.G. Vasiljevic, D.M. Todorovic, Phys. Rev. B 51, 14226 (1995)

4. D.M. Todorovic, P.M. Nikolic, Opt. Eng. 36, 432 (1997)

5. J. Bernal-Alvarado, M. Vargas, J.J. Alvarado-Gil, I. Delgadillo, A. Cruz-Orea, H. Vargas, M. TufiñoVelázquez, M.L. Albor-Aguilera, M.A. González-Trujillo, J. Appl. Phys. 83, 3807 (1998)

6. E. Martin, I. Riech, P. Diaz, J.J. Alvarado-Gil, R. Baquero, J.G. Mendoza-Alvarez, H. Vargas, A. Cruz-Orea, M. Vargas, J. Appl. Phys. 83, 2604 (1998)

7. Q. Shen, T. Toyoda, Jpn. J. Appl. Phys. 39, 3164 (2000)

8. P.M. Nikolić, D. Vasiljević-Radović, D.M. Todorović, S. Vujatović, K. Radulović, D. Urošević, S. Durić, V. Blagojević, P. Mihajlović, J. Elazar, Anal. Sci. 17, 145 (2001)

9. P.M. Nikolić, D.M. Todorović, D. Vasiljević-Radović, S. Vujatović, K. Radulović, S. Durić, V. Blagojević, D. Urošević, P. Mihajlović, J. Elazar, Anal. Sci. 17, 148 (2001)

10. D.M. Todorović, P.M. Nikolić, A.I. Bojicic, J. Elazar, M. Smiljanic, R. Petrovic, D.G. VasiljevicRadovic, K.T. Radulovic, Anal. Sci. 17, 295 (2001)

11. E. Marin, I. Reich, P. Diaz, H. Vargaz, Anal. Sci. 17, 284 (2001)

12. E. Marin, I. Reich, P. Diaz, Anal. Sci. 17, 288 (2001)

13. J. Bernal-Alvarado, M. Vargas-Luna, Anal. Sci. 17, 309 (2001)

14. K.T. Radulovic, P.M. Nikolic, D. Vasiljevic-Radovic, D.M. Toforovic, S.S. Vujatovic, A.I. Bojicic, V. Blagojevic, D. Urosevic, Rev. Sci. Instrum. 74, 595 (2003)

15. L. Bychto, M. Maliński, J. Phys. IV France 129, 213 (2005)

16. M. Maliński, L. Bychto, J. Phys. IV France 129, 237 (2005) 